Pacific Journal of Mathematics

THE WAVE FRONT SET AND THE ASYMPTOTIC SUPPORT
FOR $p$-ADIC GROUPS 


\section{THE WAVE FRONT SET AND \\ THE ASYMPTOTIC SUPPORT FOR $p$-ADIC GROUPS}

\section{TOMASZ PRZEBINDA}

We prove that for $p$-adic groups the notion of the wave front set of a representation coincides with the notion of the asymptotic support.

\section{The wave front sets of finite sums of homogeneous distributions.} Let $\Omega$ be a $p$-adic field of characteristic zero, with valuation $|\cdot|$. Let $\mathbf{g}$ be a finite dimensional vector space over $\Omega$. Fix a non-trivial character $\chi$ of the additive group $\Omega$, and a non-degenerate symmetric bilinear form $\beta$ on $\mathbf{g}$ with values in $\Omega$.

For $f \in C_{c}^{\infty}(\mathbf{g})$ (compactly supported, locally constant functions on g) define a Fourier Transform by

$$
\hat{f}(Y)=\int_{\mathbf{g}} \chi(\beta(Y, X)) f(X) d X \quad(Y \in \mathbf{g}) .
$$

Here $d X$ is a Haar measure on the additive group of $\mathbf{g}$ (normalized so that the formula $(\hat{f}) \wedge(x)=f(-x)$ holds). Then $f \rightarrow \hat{f}$ is a bijective mapping of $C_{c}^{\infty}(\mathbf{g})$ onto itself (see [Ha1] or [W, p. 107]). If $T$ is a distribution $\mathbf{g}$ then its Fourier transform $\widehat{T}$ is given by

$$
\widehat{T}(f)=T(\hat{f}) \quad\left(f \in C_{c}^{\infty}(\mathbf{g})\right) .
$$

Let $n=\operatorname{dim}_{\Omega}(\mathbf{g})$. For $f \in C_{c}^{\infty}(\mathbf{g})$ define

$$
f_{\lambda}(X)=|\lambda|^{-n} f\left(\lambda^{-1} X\right) \quad\left(X \in \mathbf{g}, \lambda \in \Omega^{\times}\right) .
$$

Fix an open subgroup $\Lambda$ of $\Omega^{x}$ with $\left[\Omega^{x}: \Lambda\right]<\infty$.

Definition 1.4. A distribution $T$ on $\mathbf{g}$ is $\Lambda$-homogeneous of degree $d \in \mathbf{C}$ if

$$
T\left(f_{\lambda}\right)=|\lambda|^{d} T(f) \quad\left(f \in C_{c}^{\infty}(\mathbf{g}), \lambda \in \Lambda\right) .
$$

Notice that

$$
\left(f_{\lambda}\right)^{-}=|\lambda|^{-n}(\hat{f})_{\lambda-1} \quad\left(f \in C_{c}^{\infty}(\mathbf{g}), \lambda \in \Omega^{x}\right),
$$

so that if $T$ is $\Lambda$-homogeneous of degree $d$ then $\widehat{T}$ is a $\Lambda$-homogeneous of degree $-n-d$. Clearly if $T$ is a function:

$$
T(f)=\int_{\mathbf{g}} T(X) f(X) d X
$$


then $T$ is $\Lambda$-homogeneous of degree $d$ iff for any $\lambda \in \Lambda$,

$$
T(\lambda X) d X=|\lambda|^{d} T(X) d X .
$$

The reader may safely focus on the case $\Lambda=\Omega^{x}$. In order to justify the generality of Definition 1.4 we mention that a distribution homogeneous with respect to a quasicharacter of $\Omega^{x}$ is $\Lambda$-homogeneous for a suitable $\Lambda$ (see for example [G-G-PS, Ch. II]).

By fixing a base of $\mathbf{g}$ we can identify it with $\Omega^{n}$ and use the norm

$$
\left|\left(\lambda, \lambda_{2}, \cdots, \lambda_{n}\right)\right|=\max \left\{\left|\lambda_{1}\right|,\left|\lambda_{2}\right|, \cdots,\left|\lambda_{n}\right|\right\} .
$$

The following simple fact will be used later.

LEMMA 1.7. Let $F$ and $V$ be open-compact subsets of g. Then there is $\delta>0$ such that for any $\lambda \in \Omega$ with $|\lambda|<\delta$ the following inclusion holds:

$$
\lambda F+V \subseteq V
$$

It is known that any compactly supported distribution on $\mathbf{g}$ has a locally constant function as a Fourier Transform.

We are going to use (1.2) to analyze the singularities of $T$ near zero.

Definition 1.8 ([He] $\S 2)$. A distribution $T$ on $\mathbf{g}$ is $\Lambda$-smooth at $Y_{0} \in \mathbf{g} \backslash\{0\}$ if there is an open neighborhood $W$ of 0 and an open neighborhood $V$ of $Y_{0}$ such that for any $f \in C_{c}^{\infty}(W)$ there is $N>0$ for which $\lambda \in \Lambda$ and $|\lambda|>N$ imply

$$
(f T)^{\wedge}(\lambda Y)=0 \text { for any } Y \in V .
$$

The complement of the set of $\Lambda$-smooth points of $T$ in $\mathbf{g} \backslash\{0\}$ is called the $\Lambda$-wave front set of $T$ at zero and is denoted $\operatorname{WF}_{\Lambda}^{0}(T)$.

The function $(f T)^{\wedge},(1.9)$, is sometimes called a localized Fourier Transform of $T$ (because $\operatorname{supp}(f T) \subseteq \operatorname{supp}(f))$. Of course this function can be expressed in terms of the convolution

$$
\begin{gathered}
(f T)^{-}=\hat{f} * \widehat{T}, \quad \text { where for } X, Y \in \mathbf{g}, \\
\hat{f} * \widehat{T}(X)=\hat{T}\left(L_{X} \hat{f}\right), \quad L_{X} \hat{f}(Y)=\hat{f}(X-Y) .
\end{gathered}
$$

Using (1.10) and the notion of a lattice in $\mathbf{g}[\mathbf{W}, \mathbf{p}$. 28] we rephrase the Definition 1.8. For a subset $U \subseteq \mathbf{g}$, let $f_{U}$ denote the characteristic function of $U$. 
LemMA 1.11. Let $T$ be a distribution on $\mathbf{g}$ and let $V$ be an opencompact subset of $\mathbf{g} \backslash\{0\}$. Then the following conditions on $V$ are equivalent:

(a) $V \cap W F_{\Lambda}^{0}(T)$ is empty.

(b) There is a lattice $U$ in $\mathbf{g}$ and a constant $c>0$, such that

$$
f_{U} * \widehat{T}(\lambda Y)=0 \text { for } \lambda \in \Lambda,|\lambda|>c, Y \in V .
$$

(c) There is a lattice $W$ in $\mathbf{g}$ and for any constant $1>\varepsilon>0 a$ constant $c_{\varepsilon}>0$ such that for any $f \in C_{c}^{\infty}(W)$,

$$
\left(f_{\gamma} T\right)^{-}(\lambda Y)=0 \text { for } \lambda, \gamma \in \Lambda,|\lambda|>c_{\varepsilon}, \varepsilon<|\gamma|<1, Y \in V .
$$

Proof. Clearly (*) implies (a). The equivalence of (a) and (b) was shown by Heifetz [He, Lemma 2.2]. We shall recall his proof to see that (b) implies (*). Let $W$ be the lattice dual to $U, f \in C_{c}^{\infty}(W)$, and let $F=-\operatorname{supp} \hat{f}$. Lemma 1.7 applied to the sets $F$ and $V$ provides a constant $\delta>0$. Put $c_{\varepsilon}=\max \left\{\delta^{-1} \varepsilon^{-1}, c\right\}$. Since by (1.5) $\operatorname{supp}\left(f_{\gamma}\right)^{\wedge}=$ $\gamma^{-1}$ supp $\hat{f}$ we see that (under the assumptions of $(*)$ )

$$
\begin{aligned}
\left(f_{\gamma} T\right)^{\wedge}(\lambda Y) & =\left(f_{\gamma} f_{W} T\right)^{\wedge}(\lambda Y) \\
& \left.=\int_{\mathbf{g}}\left(f_{\gamma}\right)^{\wedge}(Z) f_{W} T\right)^{\wedge}\left(\lambda\left(-\lambda^{-1} Z+Y\right)\right) d Z=0 .
\end{aligned}
$$

The reader may compare this proof with [Hö, 8.1.1] to see that the analogous argument in the classical situation is more complex.

Lemma 1.11 has the following immediate

COROLlaRY 1.12. The wave front set $\mathrm{WF}_{\Lambda}^{0}(T)$ contains the set $A$ of those $Y \in \mathbf{g} \backslash\{0\}$ satisfying the condition that for any lattice $U \subseteq \mathbf{g}$ and any constant $c>0$ there is $\lambda \in \lambda$ with $|\lambda|>c$ such that $f_{U} * \widehat{T}(\lambda Y) \neq 0$.

Clearly Lemma 1.11 implies that

$$
\mathrm{WF}_{\Lambda}^{0}(T) \subseteq \Lambda \cdot \operatorname{supp} \widehat{T} .
$$

Also, since for any lattice $U \subseteq \mathbf{g}$ the support of $f_{U} \widehat{T}$ is compact, the wave front set of $T$ is the same as that associated to the truncation $T_{U}$ of $T$ at infinity, defined by $\widehat{T}_{U}=\widehat{T}-f_{U} \widehat{T}$. Therefore we have another

CoROLlaRY 1.14. The wave front set $\mathrm{WF}_{\Lambda}^{0}(T)$ is contained in the set $B$, the intersection of all $\Lambda \cdot \operatorname{supp} \widehat{T}_{U}$, where $U$ varies over all lattices in $\mathbf{g}$. 
Next we define a $p$-adic analog of the classical notion of an asymptotic cone (see [Hö, 8.1.7]). For any subset $E$ of $\mathbf{g} \backslash\{0\}$ define its $\Lambda$-asymptotic cone to be the set

$$
\mathrm{AC}_{\Lambda}(E)=\left\{\lim _{j+\infty} \lambda_{j} Z_{j} \mid \lambda_{j} \in \Lambda, \lim _{j \rightarrow \infty} \lambda_{j}=0, Z_{j} \in E\right\} .
$$

By a $\Lambda$-conical subset of $\mathbf{g}$ we will mean a subset closed under multiplication by elements of $\Lambda$. Then $\mathrm{AC}_{\Lambda}(E)$ is a closed $\Lambda$-conical subset of $\mathbf{g}$.

THeORem 1.16. For any distribution $T$ on $\mathbf{g}$ define the sets $A$ and $B$ as in Corollaries 1.12 and 1.14 respectively. Then

$$
A \subseteq \mathrm{WF}_{\Lambda}^{0}(T) \subseteq B \subseteq \mathrm{AC}_{\Lambda}(\operatorname{supp} \widehat{T}) .
$$

Moreover all these sets (1.17) coincide if $T$ is $\Lambda$-homogeneous.

Proof. Only the last inclusion in (1.17) remains to be verified. It is obvious, however, if we realize that for any lattice $U$ in $\mathbf{g}$ the support of $\widehat{T}_{U}$ is contained in the intersection of the support of $\widehat{T}$ with the complement of $U$ in $\mathbf{g}$.

LEMMA 1.18. For any finite sequence of real numbers $d_{1}<d_{2}<$ $\ldots<d_{r}$ and a sequence $a_{1}, a_{2}, \ldots, a_{r}$ of complex numbers define the function

$$
F(x)=a_{1} x^{d_{1}}+a_{2} x^{d_{2}}+\cdots+a_{r} x^{d_{r}} \quad(x>0) .
$$

Then either $F$ is identically equal to zero or $F$ has at most $r-1$ zeros.

We omit the elementary proof.

Theorem 1.19. Let $T_{1}, T_{2}, \ldots, T_{r}$ be $\Lambda$-homogeneous distributions on $\mathbf{g}$ of degrees $d_{1}<d_{2}<\cdots<d_{r}$ respectively. Put $T=T_{1}+T_{2}+\cdots+$ $T_{r}$. then

$$
\mathrm{WF}_{\Lambda}^{0}(T)=\bigcup_{j=1}^{r} \mathrm{WF}_{\Lambda}^{0}\left(T_{j}\right)
$$

Proof. Since the wave front set of a finite sum of distributions is clearly contained in the union of the wave front sets of the summands, it will suffice to verify the inclusion

$$
\mathrm{WF}_{\lambda}^{0}(T) \supseteq \bigcup_{j=1}^{r} \mathrm{WF}_{\Lambda}^{0}\left(T_{j}\right)
$$


Take $V$ disjoint with $\mathrm{WF}_{\Lambda}^{0}(T)$ as in Lemma 1.11 (a). Then by (c)

$$
\begin{aligned}
& 0=\left(f_{\gamma} T\right)^{\wedge}\left(\gamma^{-1} \lambda Y\right)=\sum_{j=1}^{r}|\gamma|^{d_{j}}\left(f T_{j}\right)^{\wedge}(\lambda Y) \\
& \text { for } f \in C_{c}^{\infty}(W), \lambda \in \Lambda, \gamma \in \Lambda,|\gamma|>c_{\varepsilon}, \varepsilon<|\gamma|<1, Y \in V .
\end{aligned}
$$

Choose $\varepsilon>0$ so that there are at least $r$ elements in the set $(\varepsilon, 1] \cap$ $\{|\gamma| \mid \gamma \in \Lambda$. Then Lemma 1.18 implies that each summand in (1.21) is zero.

2. $P$-adic wave front sets of group representation. Let $\mathbf{G}$ be a connected, reductive $\Omega$-group and $G$ the subgroup of all $\Omega$-rational points in $\mathbf{G}$. Then $G$ with its usual topology is a locally compact, totally disconnected, unimodular group. Let $\mathbf{g}$ be the Lie algebra of $G$. Then $\mathbf{g}$ is a vector space over $\Omega$ of finite dimension and $G$ operates on $\mathbf{g}$ by means of the adjoint representation. Assume that the form $\beta$ in (1.1) is $G$-invariant.

Let $\pi$ be an irreducible admissible representation of $G$ and

$$
\Theta_{\pi}(f)=\operatorname{tr} \pi(f) \quad\left(f \in C_{c}^{\infty}(G)\right)
$$

be its character.

Let $N$ be the set of all elements of $\mathbf{g}$ which are nilpotent. Then $N$ is the union of a finite number of $G$-orbits which are called the nilpotent orbits. For all this see [Ha1], [Ha2]. Harish-Chandra [He 1, p. 180] has shown that one can choose an open neighborhood $U$ of zero in $\mathbf{g}$ and, for each nilpotent orbit $\mathbf{O}$, a complex constant $c_{0}$ such that

$$
\Theta_{\pi}(\exp (X))=\sum_{\mathbf{O}} c_{\mathbf{O}} \hat{\mu}_{\mathbf{O}}(X) \quad(X \in U) .
$$

Here $\mu_{0}$ is a Radon measure on $\mathbf{g}$ given by

$$
\mu_{\mathbf{O}}(f)=\int_{G / G_{0}} f\left(\operatorname{Ad} g \cdot X_{0}\right) d g^{*} \quad\left(f \in C_{c}^{\infty}(\mathbf{g})\right)
$$

where $X_{0} \in \mathbf{O}$ and $G_{0}$ is the stabilizer of $X_{0}$ in $G$ (see [R]).

It follows from Theorem 1 in $[R]$, that $\mu_{O}$ is a $\Omega^{\times}$-homogeneous distribution on $\mathbf{g}$ of degree $d=-n+\operatorname{dim}_{\Omega}(\mathbf{O}) / 2$. Therefore, via statement (1.5), $\hat{\mu}_{0}$ is a homogeneous distribution of degree $-\operatorname{dim}_{\Omega}(\mathbf{O}) / 2$.

Let $\pi$ be an admissible representation of $G$ of finite length. Put

$$
T=\Theta_{\pi} \cdot \exp \text {. }
$$

Then (2.1) implies that

$$
T=\sum_{j=1}^{r} T_{j}
$$


where the $T_{j}$ 's are homogeneous distributions on $\mathbf{g}$ of degrees $d_{j}(j=$ $1,2, \ldots, r)$. Explicitly

$$
T_{j}=\sum_{\operatorname{dim} \mathbf{O} / 2=-d_{j}} c_{\mathbf{O}} \hat{\mu}_{\mathbf{O}} .
$$

Retain the above notation. Then Theorem 1.19 implies the following

THEOREM 2.2. Let $\pi$ be an admissible representation of $G$ of finite length. Then

$$
\mathrm{WF}_{\Lambda}^{0}(T)=\bigcup_{j=1}^{r} \operatorname{supp} \widehat{T}_{j}
$$

The left hand side of the first equation may be thought of as the wave front set of the representation $\pi$ (see $[\mathrm{H}],[\mathrm{He}]$ ) and the right hand side as the asymptotic support (see [B-V]) of $\pi$. Recall also [He, Theorem 3.4] that for $\pi$ unitary $\mathrm{WF}_{\Lambda}^{0}(T)$ coincides with the wave front set of $\pi$ defined by the trace class operators. A statement analogous to Theorem 2.2 for the real reductive Lie groups was conjectured in [B-V] (and should hold via the inverse of the Lefschetz principle). Theorem 1.19 is true in the real case and its proof is equally easy.

Acknowledgment. I would like to thank Roberto Scaramuzzi for informing me about the paper [HE] of D. B. Heifetz. I am indebted to Roger Howe for the helpful revision of the original text. This text has been rewritten later by the author along the lines sketched by the referee. I would like to thank him for his suggestions.

\section{REFERENCES}

[B-V] D. Barbasch and P. Vogan, Jr., The local structure of characters, J. Funct. Anal., 37 (1980), 27-55.

[G-G-PS] I. M. Gelfand, M. I. Graev, and I. I. Pyatetskii-Shapiro, Representation theory and automorphic functions, W. B. Saunders, Philadelphia (1969).

$[\mathrm{H}] \quad$ Roger Howe, Wave front sets of representation of Lie groups, automorphic forms, representation theory, and arithmetic, Tata Inst., Bombay (1979).

[Ha1] Harish-Chandra, The Characters of Reductive p-Adic Groups, Contributions to Algebra, Academic Press, New York (1977).

[Ha2] _ Harmonic analysis on reductive p-adic groups, in Harmonic Analysis on Homogenous Spaces Amer. Math. Soc., Providence, Rhode Island, 1973.

[He] D. B. Heifetz, p-adic oscillatory integrals and wave front sets, Pacific J. Math., 116, No. 2, (1985), 285-305.

[Hö] Lars Hörmander, The Analysis of Linear Partial Differential Operators I, Springer Verlag, 1983. 
[R] R. Ranga Rao, Orbital integrals in reductive groups, Ann. of Math., 96 (1972), 505-510.

[W] André Weil, Basic Number Theory, Springer Verlag (1974).

Received February 18, 1987. Supported by NSF Grant DMS-8503781.

THE UNIVERSITY OF UTAH

Salt Lake City, UT 84112 



\section{PACIFIC JOURNAL OF MATHEMATICS EDITORS}

\author{
V. S. VARADARAJAN \\ (Managing Editor) \\ University of California \\ Los Angeles, CA 90024-1555-05 \\ Herbert Clemens \\ University of Utah \\ Salt Lake City, UT 84112 \\ THOMAs ENRIGHT \\ University of California, San Diego \\ La Jolla, CA 92093
}

R. FINN

Stanford University

Stanford, CA 94305

HermanN FlaschKa

University of Arizona

Tucson, AZ 85721

VAughan F. R. Jones

University of California

Berkeley, CA 94720

STEVEN KeRCKHOFF

Stanford University

Stanford, CA 94305

\author{
RobION KIRBY \\ University of California \\ Berkeley, CA 94720 \\ C. C. MoOre \\ University of California \\ Berkeley, CA 94720
}

Harold Stark

University of California, San Diego

La Jolla, CA 92093

\section{ASSOCIATE EDITORS
R. ARENS
E. F. BECKENBACH
B. H. NeumanN
F. WoLF
K. YoshidA (1906-1982)}

\section{SUPPORTING INSTITUTIONS}

UNIVERSITY OF ARIZONA
UNIVERSITY OF BRITISH COLUMBIA
CALIFORNIA INSTITUTE OF TECHNOLOGY
UNIVERSITY OF CALIFORNIA
MONTANA STATE UNIVERSITY
UNIVERSITY OF NEVADA, RENO
NEW MEXICO STATE UNIVERSITY
OREGON STATE UNIVERSITY

\author{
UNIVERSITY OF OREGON \\ UNIVERSITY OF SOUTHERN CALIFORNIA \\ STANFORD UNIVERSITY \\ UNIVERSITY OF HAWAII \\ UNIVERSITY OF TOKYO \\ UNIVERSITY OF UTAH \\ WASHINGTON STATE UNIVERSITY \\ UNIVERSITY OF WASHINGTON
}

The Supporting Institutions listed above contribute to the cost of publication of this Journal, but they are not owners or publishers and have no responsibility for its content or policies.

Mathematical papers intended for publication in the Pacific Journal of Mathematics should be in typed form or offset-reproduced (not dittoed), double spaced with large margins. Please do not use built up fractions in the text of the manuscript. However, you may use them in the displayed equations. Underline Greek letters in red, German in green, and script in blue. The first paragraph must be capable of being used separately as a synopsis of the entire paper. In particular it should contain no bibliographic references. Please propose a heading for the odd numbered pages of less than 35 characters. Manuscripts, in triplicate, may be sent to any one of the editors. Please classify according to the scheme of Math. Reviews, Index to Vol. 39. Supply name and address of author to whom proofs should be sent. All other communications should be addressed to the managing editor, or Elaine Barth, University of California, Los Angeles, California 90024-1555-05.

There are page-charges associated with articles appearing in the Pacific Journal of Mathematics. These charges are expected to be paid by the author's University, Government Agency or Company. If the author or authors do not have access to such Institutional support these charges are waived. Single authors will receive 50 free reprints; joint authors will receive a total of 100 free reprints. Additional copies may be obtained at cost in multiples of 50 .

The Pacific Journal of Mathematics is issued monthly as of January 1966. Regular subscription rate: $\$ 190.00$ a year (5 Vols., 10 issues). Special rate: $\$ 95.00$ a year to individual members of supporting institutions.

Subscriptions, orders for numbers issued in the last three calendar years, and changes of address should be sent to Pacific Journal of Mathematics, P.O. Box 969, Carmel Valley, CA 93924, U.S.A. Old back numbers obtainable from Kraus Periodicals Co., Route 100, Millwood, NY 10546.

The Pacific Journal of Mathematics at P.O. Box 969, Carmel Valley, CA 93924 (ISSN 0030-8730) publishes 5 volumes per year. Application to mail at Second-class postage rates is pending at Carmel Valley, California, and additional mailing offices. Postmaster: send address changes to Pacific Journal of Mathematics, P.O. Box 969, Carmel Valley, CA 93924.

\section{PUBLISHED BY PACIFIC JOURNAL OF MATHEMATICS, A NON-PROFIT CORPORATION}

Copyright (C) 1990 by Pacific Journal of Mathematics 


\section{Pacific Journal of Mathematics}

\section{Vol. 141, No. 2 December, 1990}

Ulrich F. Albrecht, Locally $A$-projective abelian groups and

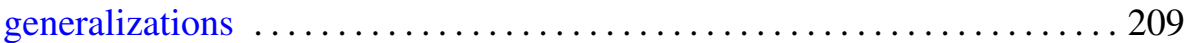

Michel Carpentier, Sommes exponentielles dont la géométrie est très belle: $p$-adic estimates ..................................... 229

G. Deferrari, Angel Rafael Larotonda and Ignacio Zalduendo, Sheaves and functional calculus

Jane M. Hawkins, Properties of ergodic flows associated to product odometers ........................................287

Anthony To-Ming Lau and Viktor Losert, Complementation of certain subspaces of $L_{\infty}(G)$ of a locally compact group ............... 295

Shahn Majid, Matched pairs of Lie groups associated to solutions of the

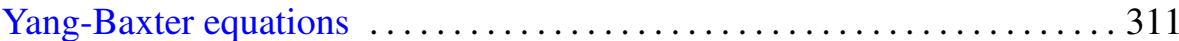

Diego Mejia and C. David (Carl) Minda, Hyperbolic geometry in $k$-convex

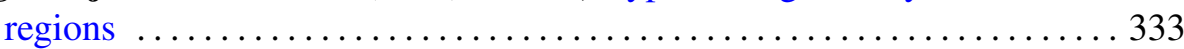

Vladimír Müller, Kaplansky's theorem and Banach PI-algebras ...........355

Raimo Näkki, Conformal cluster sets and boundary cluster sets coincide . . . 363

Tomasz Przebinda, The wave front set and the asymptotic support for $p$-adic groups

R. F. Thomas, Some fundamental properties of continuous functions and topological entropy 